Universidad Católica del Norte

Antofagasta - Chile

\title{
Some hyperstability results of a $p$-radical functional equation related to quartic mappings in non-Archimedean Banach spaces
}

\author{
Ahmed Nuino \\ University of Ibn Tofail, Morocco \\ Mustapha Esseghyr Hryrou \\ University of Ibn Tofail, Morocco \\ and \\ Samir Kabbaj \\ University of Ibn Tofail, Morocco \\ Received : August 2019. Accepted : March 2021
}

\begin{abstract}
The aim of this paper is to introduce and solve the following $p$ radical functional equation related to quartic mappings

$$
\begin{aligned}
f\left(\sqrt[p]{2 x^{p}+y^{p}}\right)+f & \left(\sqrt[p]{2 x^{p}-y^{p}}\right)=4 f\left(\sqrt[p]{x^{p}+y^{p}}\right)+4 f\left(\sqrt[p]{x^{p}-y^{p}}\right) \\
& +24 f(x)-6 f(y), \quad x, y \in \mathbf{R},
\end{aligned}
$$

where $f$ is a mapping from $\mathbf{R}$ into a vector space $X$ and $p \geq 3$ is an odd natural number. Using an analogue version of Brzdȩk's fixed point theorem [13], we establish some hyperstability results for the considered equation in non-Archimedean Banach spaces. Also, we give some hyperstability results for the inhomogeneous p-radical functional equation related to quartic mappings

$$
\begin{aligned}
f\left(\sqrt[p]{2 x^{p}+y^{p}}\right)+f & \left(\sqrt[p]{2 x^{p}-y^{p}}\right)=4 f\left(\sqrt[p]{x^{p}+y^{p}}\right)+4 f\left(\sqrt[p]{x^{p}-y^{p}}\right) \\
& +24 f(x)-6 f(y)+G(x, y) .
\end{aligned}
$$
\end{abstract}

Keywords: hyperstability, non-Archimedean Banach spaces, radical functional equations, quartic functional equations.

Mathematics Subject Classification: Primary: 39B82; Secondary: 39B62, 47H14, 47J20, 47H10. 


\section{Introduction}

A classical question in the theory of functional equation is the following: "When is it true that a function which approximately satisfies a functional equation must be close to an exact solution of this equation."

If the answer is affirmative, then we say that equation is stable. In 1940, S. M. Ulam [36]) asked the following question concerning the stability of group homomorphisms

Let $\left(G_{1}, *_{1}\right)$ be a group and let $\left(G_{2}, *_{2}\right)$ be a metric group with a metric $d(.,$.$) . Given \varepsilon>0$, does there exists a $\delta>0$ such that if a mapping $h: G_{1} \rightarrow G_{2}$ satisfies the inequality

$$
d\left(h\left(x *_{1} y\right), h(x) *_{2} h(y)\right)<\delta
$$

for all $x, y \in G_{1}$, then there exists a homomorphism $H: G_{1} \rightarrow G_{2}$ with

$$
d(h(x), H(x))<\varepsilon
$$

for all $x \in G_{1}$ ?

This question seems to be the starting point of studying the stability of functional equations. Since then, this question has attracted the attention of many researchers. The first partial answer was raised by D. H. Hyers [26] in 1941 under the assumption that $G_{1}$ and $G_{2}$ are Banach spaces for the the additive functional equation as follows:

Theorem 1.1. [26] Let $E_{1}$ and $E_{2}$ be two Banach spaces and $f: E_{1} \rightarrow E_{2}$ be a function such that

$$
\|f(x+y)-f(x)-f(y)\| \leq \delta
$$

for some $\delta>0$ and for all $x, y \in E_{1}$. Then the limit

$$
A(x)=\lim _{n \rightarrow \infty} 2^{-n} f\left(2^{n} x\right)
$$

exists for each $x \in E_{1}$, and $A: E_{1} \rightarrow E_{2}$ is the unique additive function such that

$$
\|f(x)-A(x)\| \leq \delta
$$

for all $x \in E_{1}$. Moreover, if $f(t x)$ is continuous in $t$ for each fixed $x \in E_{1}$, then the function $A$ is linear. 
Later, T. Aoki [9] and D. G. Bourgin [10] considered the problem of stability with unbounded Cauchy differences. In 1978, Th. M. Rassias [33] attempted to weaken the condition for the bound of the norm of Cauchy difference $\|f(x+y)-f(x)-f(y)\|$ and proved a generalization of Theorem 1.1 by using a direct method (cf. Theorem 1.2):

Theorem 1.2. [33] Let $E_{1}$ and $E_{2}$ be two Banach spaces. If $f: E_{1} \rightarrow E_{2}$ satisfies the inequality

$$
\|f(x+y)-f(x)-f(y)\| \leq \theta\left(\|x\|^{p}+\|y\|^{p}\right)
$$

for some $\theta \geq 0$, for some $p \in \mathbf{R}$ with $0 \leq p<1$, and for all $x, y \in E_{1}$, then there exists a unique additive function $A: E_{1} \rightarrow E_{2}$ such that

$$
\|f(x)-A(x)\| \leq \frac{2 \theta}{2-2^{p}}\|x\|^{p}
$$

for each $x \in E_{1}$. If, in addition, $f(t x)$ is continuous in $t$ for each fixed $x \in E_{1}$, then the function $A$ is linear.

After then, Th. M. Rassias [34],[35] motivated Theorem 1.2 as follows:

Theorem 1.3. [34],[35] Let $E_{1}$ be a normed space, $E_{2}$ be a Banach space, and $f: E_{1} \rightarrow E_{2}$ be a function. If $f$ satisfies the inequality

$$
\|f(x+y)-f(x)-f(y)\| \leq \theta\left(\|x\|^{p}+\|y\|^{p}\right)
$$

for some $\theta \geq 0$, for some $p \in \mathbf{R}$ with $p \neq 1$, and for all $x, y \in E_{1}-\left\{0_{E_{1}}\right\}$, then there exists a unique additive function $A: E_{1} \rightarrow E_{2}$ such that

$$
\|f(x)-A(x)\| \leq \frac{2 \theta}{\left|2-2^{p}\right|}\|x\|^{p}
$$

for each $x \in E_{1}-\left\{0_{E_{1}}\right\}$.

Note that Theorem 1.3 reduces to Theorem 1.1 when $p=0$. For $p=1$, the analogous result is not valid. Also, J. Brzdęk [12] showed the estimation (1.2) is optimal for $p \geq 0$ in the general case.

In 1994, P. Găvruţa [25] provided a further generalization of Rassias theorem in which he replaced the bound $\theta\left(\|x\|^{p}+\|y\|^{p}\right)$ in (1.1) by a general control function $\varphi(x, y)$ for the existence of a unique linear mapping. 
Recently, J. Brzdęk [16] showed that Theorem 1.3 can be significantly improved. Namely, in the case $p<0$, each $f: E_{1} \rightarrow E_{2}$ satisfying (1.1) must actually be additive. This result is called the hyperstability of Cauchy functional equation. However, the term of hyperstability was introduced for the first time probably in [30] and it was developed with the fixed point theorem of Brzdȩk in [13]. There after, the hyperstability of a several functional equations have been studied by many authors (see, for example, $[4,5,2,16,30])$.

In 2013, Brzdẹk [15] improved, extended and complemented several earlier classical stability results concerning the additive Cauchy equation (in particular Theorem 1.3). Over the last few years, many mathematicians have investigated various generalizations, extensions and applications of the Hyers-Ulam stability of a number of functional equations (see, for instance, [17], [18] and references therein).

In 2005, S. H. Lee et al [29] introduced and solve the following quartic functional equation

$f(2 x+y)+f(2 x-y)=4 f(x+y)+4 f(x-y)+24 f(x)-6 f(y) \quad x, y \in X$,

where $f: X \rightarrow Y$ with $X$ and $Y$ are two vector spaces. Namely, they proved that every solution of (1.3) is given as $f(x)=F(x, x)$, where $F: X \times X \rightarrow Y$ is a symmetric biquadratic and defined by

$$
B(x, y, z)=\frac{1}{12}(f(x+y)+f(x-y)-2 f(x)-2 f(y)) .
$$

During the $16^{\text {th }}$ International Conference on Functional Equations and Inequalities (Bȩdlewo, Poland, May 17-23, 2015), W. Sintunavarat presented a talk concerning the Ulam type stability (for information and further references concerning this notion see, e.g., [11]) of the so-called radical functional equation

$$
f\left(\sqrt{x^{2}+y^{2}}\right)=f(x)+f(y)
$$

in the class of real functions. A question of J. Schwaiger about the general solution of the equation was answered a bit later by the author of this paper 
(see [32], p. 196). In this regard, many papers concerning the solutions and stability of radical functional equations have been established (the reader can refer, for example, to $[6,7,8,22,21,27,28])$.

Let us recall (see, for instance, [27]) some basic definitions and facts concerning non-Archimedean normed spaces.

Definition 1.4. By a non-Archimedean field we mean a field $\mathbf{K}$ equipped with a function (valuation) $|\cdot|: \mathbf{K} \rightarrow[0, \infty)$ such that for all $r, s \in \mathbf{K}$, the following conditions hold:

1. $|r|=0$ if and only if $r=0$,

2. $|r s|=|r||s|$,

3. $|r+s| \leq \max \{|r|,|s|\}$.

The pair $(\mathbf{K},||$.$) is called a valued field.$

In any non-Archimedean field we have $|1|=|-1|=1$ and $|n| \leq 1$ for $n \in \mathbf{N}_{0}$. In any field $\mathbf{K}$ the function $|\cdot|: \mathbf{K} \rightarrow \mathbf{R}_{+}$given by

$$
|x|:= \begin{cases}0, & x=0 \\ 1, & x \neq 0\end{cases}
$$

is a valuation which is called trivial, but the most important examples of non-Archimedean fields are $p$-adic numbers which have gained the interest of physicists for their research in some problems coming from quantum physics, $p$-adic strings and superstrings.

Definition 1.5. Let $X$ be a vector space over a scalar field $\mathbf{K}$ with a nonArchimedean non-trivial valuation $|\cdot|$. A function $\|\cdot\|_{*}: X \rightarrow \mathbf{R}$ is a non-Archimedean norm (valuation) if it satisfies the following conditions:

1. $\|x\|_{*}=0$ if and only if $x=0$,

2. $\|r x\|_{*}=|r|\|x\|_{*}(r \in \mathbf{K}, x \in X)$,

3. The strong triangle inequality (ultrametric); namely : $\|x+y\|_{*} \leq \max \left\{\|x\|_{*},\|y\|_{*}\right\} \quad x, y \in X$

Then $\left(X,\|\cdot\|_{*}\right)$ is called a non-Archimedean normed space or an ultrametric normed space. 
Definition 1.6. Let $\left\{x_{n}\right\}$ be a sequence in a non-Archimedean normed space $X$.

1. A sequence $\left\{x_{n}\right\}_{n=1}^{\infty}$ in a non-Archimedean space is a Cauchy sequence if the sequence $\left\{x_{n+1}-x_{n}\right\}_{n=1}^{\infty}$ converges to zero;

2. The sequence $\left\{x_{n}\right\}$ is said to be convergent if, there exists $x \in X$ such that, for any $\varepsilon>0$, there is a positive integer $N$ such that $\left\|x_{n}-x\right\|_{*} \leq \varepsilon$, for all $n \geq N$. Then the point $x \in X$ is called the limit of the sequence $\left\{x_{n}\right\}$, which is denoted by $\lim _{n \rightarrow \infty} x_{n}=x$;

3. If every Cauchy sequence in $X$ converges, then the non-Archimedean normed space $X$ is called a non-Archimedean Banach space or an non-Archimedean Banach space.

Throughout this paper, we will denote the set of natural numbers by $\mathbf{N}, \mathbf{N}_{0}:=\mathbf{N} \cup\{0\}$, the set of real numbers by $\mathbf{R}, \mathbf{R}_{+}=[0, \infty)$ the set of non negative real numbers and $\mathbf{R}_{0}=\mathbf{R} \backslash\{0\}$. By $\mathbf{N}_{m 0}, m_{0} \in \mathbf{N}$, we will denote the set of all natural numbers greater than or equal to $m_{0}$.

Let $X$ be a linear space and let $p \in \mathbf{N}_{3}$ be an odd natural number. We introduce the following functional equation

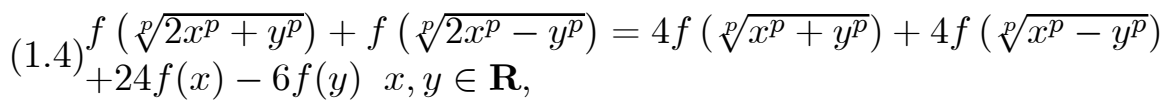

where $f: \mathbf{R} \rightarrow X$ which is called $p$-radical functional equation related to quartic equation (1.3).

The main purpose of this paper is to achieve the general solution of the functional equation (1.4) and establish some hyperstability results for the considered equation in non-Archimedean Banach space. We also provide some corollaries and outcomes concerning the hyperstability results for the inhomogeneous of $p$-radical functional equation.

Before proceeding to the main results, we state Theorem 1.7 which is useful for our purpose. To present it, we introduce the following three hypotheses:

(H1) $X$ is a nonempty set, $Y$ is an non-Archimedean Banach space over a non-Archimedean field, $f_{1}, \ldots, f_{k}: X \longrightarrow X$ and $L_{1}, \ldots, L_{k}: X \longrightarrow$ $\mathbf{R}_{+}$are given. 
(H2) $\mathcal{T}: Y^{X} \longrightarrow Y^{X}$ is an operator satisfying the inequality $\| \mathcal{T} \xi(x)-$ $\mathcal{T} \mu(x) \|_{*} \leq \max _{1 \leq i \leq k}\left\{L_{i}(x)\left\|\xi\left(f_{i}(x)\right)-\mu\left(f_{i}(x)\right)\right\|_{*}\right\}, \xi, \mu \in Y^{X}, x \in$ $X$.

(H3) $\Lambda: \mathbf{R}_{+}^{X} \longrightarrow \mathbf{R}_{+}^{X}$ is a linear operator defined by

$$
\Lambda \delta(x):=\max _{1 \leq i \leq k}\left\{L_{i}(x) \delta\left(f_{i}(x)\right)\right\}, \delta \in \mathbf{R}_{+}^{X}, x \in X .
$$

Thanks to a result due to J. Brzdẹk and K. Ciepliński [14, Remark 2], we state an analogue of the fixed point theorem [14, Theorem 1] in nonArchimedean Banach space. We use it to assert the existence of a unique fixed point of operator $\mathcal{T}: Y^{X} \longrightarrow Y^{X}$.

Theorem 1.7. Let hypotheses (H1)-(H3) be valid and functions $\varepsilon: X \longrightarrow$ $\mathbf{R}_{+}$and $\varphi: X \longrightarrow Y$ fulfil the following two conditions

$$
\begin{gathered}
\|\mathcal{T} \varphi(x)-\varphi(x)\|_{*} \leq \varepsilon(x), \quad x \in X, \\
\lim _{n \rightarrow \infty} \Lambda^{n} \varepsilon(x)=0, \quad x \in X .
\end{gathered}
$$

Then there exists a unique fixed point $\psi \in Y^{X}$ of $\mathcal{T}$ with

$$
\|\varphi(x)-\psi(x)\|_{*} \leq \sup _{n \in \mathbf{N}_{0}} \Lambda^{n} \varepsilon(x), \quad x \in X .
$$

Moreover

$$
\psi(x):=\lim _{n \rightarrow \infty} \mathcal{T}^{n} \varphi(x), \quad x \in X
$$

\section{Main results}

The next theorem can be derived from ([20], Corollary 2.3 and Proposition 2.4(a)). However, for the convenience of readers we present it with a direct proof.

Theorem 2.1. Let $X$ be a linear space and $p \in \mathbf{N}_{3}$ be an odd natural number. A function $f: \mathbf{R} \rightarrow X$ is a solution of the functional equation (1.4) if and only if $f(x)=Q\left(x^{p}\right)$ for all $x \in \mathbf{R}$, where $Q$ is a solution of the quartic functional equation (1.3). 
Proof. It's not hard to see that if $f(x)=Q\left(x^{p}\right)$, then $f$ is a solution of the equation (1.4). On the other hand, if $f$ is a solution of the equation (1.4), then, for each $x, y \in \mathbf{R}$, we have

$$
\begin{aligned}
Q(2 x+y)+Q(2 x-y) & =f\left(\sqrt[p]{2 \sqrt[p]{x^{p}}+\sqrt[p]{y^{p}}}\right)+f\left(\sqrt[p]{2 \sqrt[p]{x^{p}}-\sqrt[p]{y^{p}}}\right) \\
& =4 f\left(\sqrt[p]{\sqrt[p]{x^{p}}+\sqrt[p]{y^{p}}}\right)+4 f\left(\sqrt[p]{\sqrt[p]{x^{p}}-\sqrt[p]{y^{p}}}\right) \\
& +24 f(\sqrt[p]{x})-6 f(\sqrt[p]{y}) \\
& =4 Q(x+y)+4 Q(x-y)+24 Q(x)-6 Q(y) .
\end{aligned}
$$

Next, we examine the hyperstability of the equation (1.4) in non-Archimedean Banach space by using, as a basic tool, the fixed point Theorem 1.7.

Theorem 2.2. Let $p$ be an odd natural number, $\left(X,\|\cdot\|_{*}\right)$ be a nonArchimedean Banach space and let $h_{1}, h_{2}: \mathbf{R}_{0} \rightarrow \mathbf{R}_{+}$be two functions such that

$$
\begin{aligned}
\mathcal{U}:= & \left\{n \in \mathbf{N}: \alpha_{n}=\max \left\{\lambda_{1}(3 n+2) \lambda_{2}(3 n+2), \lambda_{1}(-n) \lambda_{2}(-n),\right.\right. \\
& \lambda_{1}(n+1) \lambda_{2}(n+1), \\
& \left.\left.\lambda_{1}(4 n+3) \lambda_{2}(4 n+3), \lambda_{1}(2 n+1) \lambda_{2}(2 n+1)\right\}<1\right\} \neq \phi,
\end{aligned}
$$

where

$$
\lambda_{i}(m):=\inf \left\{t \in \mathbf{R}_{+}: h_{i}(m x) \leq t h_{i}(x), \quad x \in \mathbf{R}_{0}\right\},
$$

for all $m \in \mathbf{N}$, where $i=1,2$ such that

$$
\lim _{m \rightarrow \infty} \lambda_{1}(m+1) \lambda_{2}(2 m+1)=0
$$

Assume that $f: \mathbf{R} \rightarrow X$ satisfies the inequality

$$
\begin{aligned}
& \| f\left(\sqrt[p]{2 x^{p}+y^{p}}\right)+f\left(\sqrt[p]{2 x^{p}-y^{p}}\right)-4 f\left(\sqrt[p]{x^{p}+y^{p}}\right)-4 f\left(\sqrt[p]{x^{p}-y^{p}}\right) \\
& -24 f(x)+6 f(y) \|_{*} \leq h_{1}\left(x^{p}\right) h_{2}\left(y^{p}\right),
\end{aligned}
$$

for all $x, y \in \mathbf{R}_{0}$ such that $x \neq y, \quad x \neq-y, \quad y \neq \sqrt[p]{2} x$ and $y \neq-\sqrt[p]{2} x$. Then $f$ is a solution of the equation (1.4) on $\mathbf{R}_{0}$.

Proof. Replacing $x$ by $\sqrt[p]{m+1} x$ and $y$ by $\sqrt[p]{2 m+1} x$ in the inequality (2.2), we get

$$
\begin{aligned}
& \| 4 f(\sqrt[p]{3 m+2} x)+4 f(\sqrt[p]{-m} x)+24 f(\sqrt[p]{m+1} x)-f(\sqrt[p]{4 m+3} x) \\
& -6 f(\sqrt[p]{2 m+1} x)-f(x) \|_{*} \leq h_{1}\left((m+1) x^{p}\right) h_{2}\left((2 m+1) x^{p}\right),
\end{aligned}
$$


for all $x \in \mathbf{R}_{0}$. For each $m \in \mathbf{N}$, we define the operator $\mathcal{T}_{m}: X^{\mathbf{R}_{0}} \rightarrow X^{\mathbf{R}_{0}}$ by

$$
\begin{gathered}
\mathcal{T}_{m} \xi(x):=4 \xi(\sqrt[p]{3 m+2} x)+4 \xi(\sqrt[p]{-m} x)+24 \xi(\sqrt[p]{m+1} x)-\xi(\sqrt[p]{4 m+3} x) \\
-6 \xi(\sqrt[p]{2 m+1} x),
\end{gathered}
$$

for all $\xi \in X^{\mathbf{R}_{0}}, x \in \mathbf{R}_{0}$ and the function $\varepsilon_{m}: \mathbf{R}_{0} \rightarrow \mathbf{R}_{+}$by

$$
\varepsilon_{m}(x):=h_{1}\left((m+1) x^{p}\right) h_{2}\left((2 m+1) x^{p}\right), \quad m \in \mathbf{N}, \quad x \in \mathbf{R}_{0} .
$$

We observe that

$$
\varepsilon_{m}(x) \leq \lambda_{1}(m+1) \lambda_{2}(2 m+1) h_{1}\left(x^{p}\right) h_{2}\left(x^{p}\right),
$$

for all $x \in \mathbf{R}_{0}$ and all $m \in \mathcal{U}$. Then the inequality (2.3) become as

$$
\left\|\mathcal{T}_{m} f(x)-f(x)\right\|_{*} \leq \varepsilon_{m}(x), \quad x \in \mathbf{R}_{0} .
$$

Furthermore, the operator $\Lambda_{m}: \mathbf{R}_{+}^{\mathbf{R}_{0}} \rightarrow \mathbf{R}_{+}^{\mathbf{R}_{0}}$ defined by

$$
\Lambda_{m} \delta(x):=\max _{1 \leq i \leq 5}\left\{L_{i}(x) \delta\left(f_{i}(x)\right\},\right.
$$

for all $x \in \mathbf{R}_{0}$ and all $\delta \in \mathbf{R}_{+}^{\mathbf{R}_{0}}$ where $f_{1}(x)=\sqrt[p]{3 m+2} x, f_{2}(x)=$ $\sqrt[p]{-m} x, f_{3}(x)=\sqrt[p]{m+1} x, f_{4}(x)=\sqrt[p]{4 m+3} x, f_{5}(x)=\sqrt[p]{2 m+1} x$, and $L_{1}(x)=L_{2}(x)=L_{3}(x)=L_{4}(x)=L_{5}(x)=1$.

Moreover, for every $x \in \mathbf{R}_{0}, \xi, \mu \in X^{\mathbf{R}_{0}}$, we obtain 


$$
\begin{aligned}
& \left\|\mathcal{T}_{m} \xi(x)-\mathcal{T}_{m} \mu(x)\right\|_{*} \\
& =\| 4(\xi(\sqrt[p]{3 m+2} x)-\mu(\sqrt[p]{3 m+2} x))+4(\xi(\sqrt[p]{-m} x)-\mu(\sqrt[p]{-m} x)) \\
& +24(\xi(\sqrt[p]{m+1} x)-\mu(\sqrt[p]{m+1} x))-(\xi(\sqrt[p]{4 m+3} x)-\mu(\sqrt[p]{4 m+3} x)) \\
& -6(\xi(\sqrt[p]{2 m+1} x)-\mu(\sqrt[p]{2 m+1} x)) \|_{*} \\
& \leq \max \left\{4\|\xi(\sqrt[p]{3 m+2} x)-\mu(\sqrt[p]{3 m+2} x)\|_{*}, 4\|\xi(\sqrt[p]{-m} x)-\mu(\sqrt[p]{-m} x)\|_{*},\right. \\
& 24\|\xi(\sqrt[p]{m+1} x)-\mu(\sqrt[p]{m+1} x)\|_{*},\|\xi(\sqrt[p]{4 m+3} x)-\mu(\sqrt[p]{4 m+3} x)\|_{*}, \\
& \left.6\|\xi(\sqrt[p]{2 m+1} x)-\mu(\sqrt[p]{2 m+1} x)\|_{*}\right\} \\
& \leq \max \left\{\|\xi(\sqrt[p]{3 m+2} x)-\mu(\sqrt[p]{3 m+2} x)\|_{*},\|\xi(\sqrt[p]{-m} x)-\mu(\sqrt[p]{-m} x)\|_{*},\right. \\
& \|\xi(\sqrt[p]{m+1} x)-\mu(\sqrt[p]{m+1} x)\|_{*},\|\xi(\sqrt[p]{4 m+3} x)-\mu(\sqrt[p]{4 m+3} x)\|_{*}, \\
& \left.\|\xi(\sqrt[p]{2 m+1} x)-\mu(\sqrt[p]{2 m+1} x)\|_{*}\right\} \\
& =\max _{1 \leq i \leq 5}\left\{L_{i}(x)\left\|\xi\left(f_{i}(x)\right)-\mu\left(f_{i}(x)\right)\right\|_{*}\right\}
\end{aligned}
$$

which means that (H2) is valid. Now we will show, by induction on $n \in \mathbf{N}_{0}$, that

$$
\Lambda^{n} \varepsilon_{m}(x) \leq \lambda_{1}(m+1) \lambda_{2}(2 m+1) \alpha_{m}^{n} h_{1}\left(x^{p}\right) h_{2}\left(x^{p}\right) .
$$

for all $x \in \mathbf{R}_{0}$ and all $m \in \mathcal{U}$ where

$$
\begin{gathered}
\alpha_{m}=\max \left\{\lambda_{1}(3 m+2) \lambda_{2}(3 m+2), \lambda_{1}(-m) \lambda_{2}(-m), \lambda_{1}(m+1) \lambda_{2}(m+1),\right. \\
\left.\lambda_{1}(4 m+3) \lambda_{2}(4 m+3), \lambda_{1}(2 m+1) \lambda_{2}(2 m+1)\right\} .
\end{gathered}
$$

For $n=0$, the inequality (2.5) is exactly (2.4). Next we will assume that (2.5) holds for $n=k$, where $k \in \mathbf{N}$. Then

$$
\begin{aligned}
& \Lambda_{m}^{k+1} \varepsilon_{m}(x)=\Lambda_{m}\left(\Lambda_{m}^{k} \varepsilon_{m}(x)\right) \\
& =\max \left\{\Lambda_{m}^{k} \varepsilon_{m}(\sqrt[p]{3 m+2} x), \Lambda_{m}^{k} \varepsilon_{m}(\sqrt[p]{-m} x), \Lambda_{m}^{k} \varepsilon_{m}(\sqrt[p]{m+1} x),\right. \\
& \left.\Lambda_{m}^{k} \varepsilon_{m}(\sqrt[p]{4 m+3} x), \Lambda_{m}^{k} \varepsilon_{m}(\sqrt[p]{2 m+1} x)\right\} \\
& \leq \lambda_{1}(m+1) \lambda_{2}(2 m+1) \alpha_{m}^{k} \max \left\{h_{1}\left((3 m+2) x^{p}\right) h_{2}\left((3 m+2) x^{p}\right),\right. \\
& h_{1}\left((-m) x^{p}\right) h_{2}\left((-m) x^{p}\right), h_{1}\left((m+1) x^{p}\right) h_{2}\left((m+1) x^{p}\right), \\
& \left.h_{1}\left((4 m+3) x^{p}\right) h_{2}\left((4 m+3) x^{p}\right), h_{1}\left((2 m+1) x^{p}\right) h_{2}\left((2 m+1) x^{p}\right) ;\right\} \\
& \leq \lambda_{1}(m+1) \lambda_{2}(2 m+1) \alpha_{m}^{k} \max \left\{\lambda_{1}(3 m+2) \lambda_{2}(3 m+2),\right. \\
& \lambda_{1}(-m) \lambda_{2}(-m), \lambda_{1}(m+1) \lambda_{2}(m+1), \\
& \left.\lambda_{1}(4 m+3) \lambda_{2}(4 m+3), \lambda_{1}(2 m+1) \lambda_{2}(2 m+1)\right\} h_{1}\left(x^{p}\right) h_{2}\left(x^{p}\right) \\
& =\lambda_{1}(m+1) \lambda_{2}(2 m+1) \alpha_{m}^{k+1} h_{1}\left(x^{p}\right) h_{2}\left(x^{p}\right),
\end{aligned}
$$


for all $x \in \mathbf{R}_{0}$ and all $m \in \mathcal{U}$. It shows that (2.5) holds for $n=k+1$. We conclude that the inequality (2.5) holds for all $n \in \mathbf{N}_{0}$.

Since $\alpha_{m}<1$ for all $m \in \mathcal{U}$, we get

$$
\lim _{n \rightarrow \infty} \Lambda^{n} \varepsilon_{m}(x)=0
$$

for all $x \in \mathbf{R}_{0}$. According to Theorem 1.7, there exists, for each $m \in \mathcal{U}$, a fixed point $\mathcal{F}_{m}: \mathbf{R}_{0} \rightarrow X$ of the operator $\mathcal{T}_{m}$ such that

$$
\begin{aligned}
\left\|f(x)-\mathcal{F}_{m}(x)\right\|_{*} & \leq \sup _{n \in \mathbf{N}}\left\{\begin{array}{l}
\left.\Lambda_{m}^{n} \varepsilon_{m}(x)\right\} \\
\leq
\end{array}\right. \\
& \sup _{n \in \mathbf{N}}\left\{\lambda_{1}(m+1) \lambda_{2}(2 m+1) \mathbf{R}_{0}^{n} h_{1}\left(x^{p}\right) h_{2}\left(x^{p}\right)\right\},
\end{aligned}
$$

Moreover,

$$
\mathcal{F}_{m}(x)=\lim _{n \rightarrow \infty}\left(\mathcal{T}_{m}^{n} f\right)(x), \quad x \in \mathbf{R}_{0}
$$

Next, we should prove the following inequality

$$
\begin{aligned}
& \| \mathcal{T}_{m}^{n} f\left(\sqrt[p]{2 x^{p}+y^{p}}\right)+\mathcal{T}_{m}^{n} f\left(\sqrt[p]{2 x^{p}-y^{p}}\right)-4 \mathcal{T}_{m}^{n} f\left(\sqrt[p]{x^{p}+y^{p}}\right) \\
& -4 \mathcal{T}_{m}^{n} f\left(\sqrt[p]{x^{p}-y^{p}}\right)-24 \mathcal{T}_{m}^{n} f(x)-6 \mathcal{T}_{m}^{n} f(y) \|_{*} \leq \alpha_{m}^{n} h_{1}\left(x^{p}\right) h_{2}\left(y^{p}\right),
\end{aligned}
$$

for all $m \in \mathcal{U}$ and all $x, y \in \mathbf{R}_{0}$ such that $x \neq y, x \neq-y, \quad y \neq \sqrt[p]{2} x$, $y \neq-\sqrt[p]{2} x, n \in \mathbf{N}$.

We proceed by induction that the case $n=0$ gives us (2.2). Assume that (2.7) holds for $n=k$ where $k \in \mathbf{N}$. Then for each $m \in \mathcal{U}$ and every $x, y \in \mathbf{R}_{0}$ such that $x \neq y, x \neq-y, y=\neq \sqrt[p]{2} x, y \neq-\sqrt[p]{2} x$, we have 


$$
\begin{aligned}
& \| \mathcal{T}_{m}^{k+1} f\left(\sqrt[p]{2 x^{p}+y^{p}}\right)+\mathcal{T}_{m}^{k+1} f\left(\sqrt[p]{2 x^{p}-y^{p}}\right)-4 \mathcal{T}_{m}^{k+1} f\left(\sqrt[p]{x^{p}+y^{p}}\right) \\
& -4 \mathcal{T}_{m}^{k+1} f\left(\sqrt[p]{x^{p}-y^{p}}\right)-24 \mathcal{T}_{m}^{k+1} f(x)+6 \mathcal{T}_{m}^{k+1} f(y) \|_{*} \\
& =\| 4 \mathcal{T}_{m}^{k} f\left(\sqrt[p]{3 m+2} \sqrt[p]{2 x^{p}+y^{p}}\right)+4 \mathcal{T}_{m}^{k} f\left(\sqrt[p]{-m} \sqrt[p]{2 x^{p}+y^{p}}\right) \\
& +24 \mathcal{T}_{m}^{k} f\left(\sqrt[p]{m+1} \sqrt[p]{2 x^{p}+y^{p}}\right)-\mathcal{T}_{m}^{k} f\left(\sqrt[p]{4 m+3} \sqrt[p]{2 x^{p}+y^{p}}\right) \\
& -6 \mathcal{T}_{m}^{k} f\left(\sqrt[p]{2 m+1} \sqrt[p]{2 x^{p}+y^{p}}\right)+4 \mathcal{T}_{m}^{k} f\left(\sqrt[p]{3 m+2} \sqrt[p]{2 x^{p}-y^{p}}\right) \\
& +4 \mathcal{T}_{m}^{k} f\left(\sqrt[p]{-m} \sqrt[p]{2 x^{p}-y^{p}}\right)+24 \mathcal{T}_{m}^{k} f\left(\sqrt[p]{m+1} \sqrt[p]{2 x^{p}-y^{p}}\right) \\
& -\mathcal{T}_{m}^{k} f\left(\sqrt[p]{4 m+3} \sqrt[p]{2 x^{p}-y^{p}}\right)-6 \mathcal{T}_{m}^{k} f\left(\sqrt[p]{2 m+1} \sqrt[p]{2 x^{p}-y^{p}}\right) \\
& -16 \mathcal{T}_{m}^{k} f\left(\sqrt[p]{3 m+2} \sqrt[p]{x^{p}+y^{p}}\right)-16 \mathcal{T}_{m}^{k} f\left(\sqrt[p]{-m} \sqrt[p]{x^{p}+y^{p}}\right) \\
& -96 \mathcal{T}_{m}^{k} f\left(\sqrt[p]{m+1} \sqrt[p]{x^{p}+y^{p}}\right)+4 \mathcal{T}_{m}^{k} f\left(\sqrt[p]{4 m+3} \sqrt[p]{x^{p}+y^{p}}\right) \\
& +24 \mathcal{T}_{m}^{k} f\left(\sqrt[p]{2 m+1} \sqrt[p]{x^{p}+y^{p}}\right)-16 \mathcal{T}_{m}^{k} f\left(\sqrt[p]{3 m+2} \sqrt[p]{x^{p}-y^{p}}\right) \\
& -16 \mathcal{T}_{m}^{k} f\left(\sqrt[p]{-m} \sqrt[p]{x^{p}-y^{p}}\right)-96 \mathcal{T}_{m}^{k} f\left(\sqrt[p]{m+1} \sqrt[p]{x^{p}-y^{p}}\right) \\
& +4 \mathcal{T}_{m}^{k} f\left(\sqrt[p]{4 m+3} \sqrt[p]{x^{p}-y^{p}}\right)+24 \mathcal{T}_{m}^{k} f\left(\sqrt[p]{2 m+1} \sqrt[p]{x^{p}-y^{p}}\right) \\
& -96 \mathcal{T}_{m}^{k} f(\sqrt[p]{3 m+2} x)-96 \mathcal{T}_{m}^{k} f(\sqrt[p]{-m} x)-576 \mathcal{T}_{m}^{k} f(\sqrt[p]{m+1} x) \\
& +24 \mathcal{T}_{m}^{k} f(\sqrt[p]{4 m+3} x)+144 \mathcal{T}_{m}^{k} f(\sqrt[p]{2 m+1} x)+24 \mathcal{T}_{m}^{k} f(\sqrt[p]{3 m+2} y) \\
& +24 \mathcal{T}_{m}^{k} f(\sqrt[p]{-m} y)+144 \mathcal{T}_{m}^{k} f(\sqrt[p]{m+1} y)-6 \mathcal{T}_{m}^{k} f(\sqrt[p]{4 m+3} y) \\
& -36 \mathcal{T}_{m}^{k} f(\sqrt[p]{2 m+1} y) \|_{*} \leq \max \left\{4 \| \mathcal{T}_{m}^{k} f\left(\sqrt[p]{3 m+2} \sqrt[p]{2 x^{p}+y^{p}}\right)\right. \\
& +\mathcal{T}_{m}^{k} f\left(\sqrt[p]{3 m+2} \sqrt[p]{2 x^{p}-y^{p}}\right)-4 \mathcal{T}_{m}^{k} f\left(\sqrt[p]{3 m+2} \sqrt[p]{x^{p}+y^{p}}\right) \\
& -4 \mathcal{T}_{m}^{k} f\left(\sqrt[p]{3 m+2} \sqrt[p]{x^{p}-y^{p}}\right)-24 \mathcal{T}_{m}^{k} f(\sqrt[p]{3 m+2} x)+6 \mathcal{T}_{m}^{k} f(\sqrt[p]{3 m+2} y) \|_{*}, \\
& 4 \mid \mathcal{T}_{m}^{k} f\left(\sqrt[p]{-m} \sqrt[p]{2 x^{p}+y^{p}}\right)+\mathcal{T}_{m}^{k} f\left(\sqrt[p]{-m} \sqrt[p]{2 x^{p}-y^{p}}\right)-4 \mathcal{T}_{m}^{k} f\left(\sqrt[p]{-m} \sqrt[p]{x^{p}+y^{p}}\right) \\
& -4 \mathcal{T}_{m}^{k} f\left(\sqrt[p]{-m} \sqrt[p]{x^{p}-y^{p}}\right)-24 \mathcal{T}_{m}^{k} f(\sqrt[p]{-m} x)+6 \mathcal{T}_{m}^{k} f(\sqrt[p]{-m} y) \|_{*}, \\
& 24 \| \mathcal{T}_{m}^{k} f\left(\sqrt[p]{m+1} \sqrt[p]{2 x^{p}+y^{p}}\right)+\mathcal{T}_{m}^{k} f\left(\sqrt[p]{m+1} \sqrt[p]{2 x^{p}-y^{p}}\right) \\
& -4 \mathcal{T}_{m}^{k} f\left(\sqrt[p]{m+1} \sqrt[p]{x^{p}+y^{p}}\right)-4 \mathcal{T}_{m}^{k} f\left(\sqrt[p]{m+1} \sqrt[p]{x^{p}-y^{p}}\right)-24 \mathcal{T}_{m}^{k} f(\sqrt[p]{m+1} x) \\
& +6 \mathcal{T}_{m}^{k} f(\sqrt[p]{m+1} y)\left\|_{*},\right\| \mathcal{T}_{m}^{k} f\left(\sqrt[p]{4 m+3} \sqrt[p]{2 x^{p}+y^{p}}\right) \\
& +\mathcal{T}_{m}^{k} f\left(\sqrt[p]{4 m+3} \sqrt[p]{2 x^{p}-y^{p}}\right)-4 \mathcal{T}_{m}^{k} f\left(\sqrt[p]{4 m+3} \sqrt[p]{x^{p}+y^{p}}\right)-4 \mathcal{T}_{m}^{k} f\left(\sqrt[p]{4 m+3} \sqrt[p]{x^{p}-y^{p}}\right) \\
& -24 \mathcal{T}_{m}^{k} f(\sqrt[p]{4 m+3} x)+6 \mathcal{T}_{m}^{k} f(\sqrt[p]{4 m+3} y) \|_{*}, \\
& 6 \| \mathcal{T}_{m}^{k} f\left(\sqrt[p]{2 m+1} \sqrt[p]{2 x^{p}+y^{p}}\right)+\mathcal{T}_{m}^{k} f\left(\sqrt[p]{2 m+1} \sqrt[p]{2 x^{p}-y^{p}}\right)-4 \mathcal{T}_{m}^{k} f\left(\sqrt[p]{2 m+1} \sqrt[p]{x^{p}+y^{p}}\right) \\
& \left.-4 \mathcal{T}_{m}^{k} f\left(\sqrt[p]{2 m+1} \sqrt[p]{x^{p}-y^{p}}\right)-24 \mathcal{T}_{m}^{k} f(\sqrt[p]{2 m+1} x)+6 \mathcal{T}_{m}^{k} f(\sqrt[p]{2 m+1} y) \|_{*}\right\}
\end{aligned}
$$




$$
\begin{aligned}
& \leq \max \left\{\| \mathcal{T}_{m}^{k} f\left(\sqrt[p]{3 m+2} \sqrt[p]{2 x^{p}+y^{p}}\right)+\mathcal{T}_{m}^{k} f\left(\sqrt[p]{3 m+2} \sqrt[p]{2 x^{p}-y^{p}}\right)\right. \\
& -4 \mathcal{T}_{m}^{k} f\left(\sqrt[p]{3 m+2} \sqrt[p]{x^{p}+y^{p}}\right) \\
& -4 \mathcal{T}_{m}^{k} f\left(\sqrt[p]{3 m+2} \sqrt[p]{x^{p}-y^{p}}\right)-24 \mathcal{T}_{m}^{k} f(\sqrt[p]{3 m+2} x)+6 \mathcal{T}_{m}^{k} f(\sqrt[p]{3 m+2} y) \|_{*}, \\
& \| \mathcal{T}_{m}^{k} f\left(\sqrt[p]{-m} \sqrt[p]{2 x^{p}+y^{p}}\right)+\mathcal{T}_{m}^{k} f\left(\sqrt[p]{-m} \sqrt[p]{2 x^{p}-y^{p}}\right)-4 \mathcal{T}_{m}^{k} f\left(\sqrt[p]{-m} \sqrt[p]{x^{p}+y^{p}}\right) \\
& -4 \mathcal{T}_{m}^{k} f\left(\sqrt[p]{-m} \sqrt[p]{x^{p}-y^{p}}\right)-24 \mathcal{T}_{m}^{k} f(\sqrt[p]{-m} x)+6 \mathcal{T}_{m}^{k} f(\sqrt[p]{-m} y) \|_{*}, \\
& \| \mathcal{T}_{m}^{k} f\left(\sqrt[p]{m+1} \sqrt[p]{2 x^{p}+y^{p}}\right)+\mathcal{T}_{m}^{k} f\left(\sqrt[p]{m+1} \sqrt[p]{2 x^{p}-y^{p}}\right)-4 \mathcal{T}_{m}^{k} f\left(\sqrt[p]{m+1} \sqrt[p]{x^{p}+y^{p}}\right) \\
& -4 \mathcal{T}_{m}^{k} f\left(\sqrt[p]{m+1} \sqrt[p]{x^{p}-y^{p}}\right)-24 \mathcal{T}_{m}^{k} f(\sqrt[p]{m+1} x)+6 \mathcal{T}_{m}^{k} f(\sqrt[p]{m+1} y) \|_{*}, \\
& \| \mathcal{T}_{m}^{k} f\left(\sqrt[p]{4 m+3} \sqrt[p]{2 x^{p}+y^{p}}\right)+\mathcal{T}_{m}^{k} f\left(\sqrt[p]{4 m+3} \sqrt[p]{2 x^{p}-y^{p}}\right)-4 \mathcal{T}_{m}^{k} f\left(\sqrt[p]{4 m+3} \sqrt[p]{x^{p}+y^{p}}\right) \\
& -4 \mathcal{T}_{m}^{k} f\left(\sqrt[p]{4 m+3} \sqrt[p]{x^{p}-y^{p}}\right)-24 \mathcal{T}_{m}^{k} f(\sqrt[p]{4 m+3} x)+6 \mathcal{T}_{m}^{k} f(\sqrt[p]{4 m+3} y) \|_{*}, \\
& \| \mathcal{T}_{m}^{k} f\left(\sqrt[p]{2 m+1} \sqrt[p]{2 x^{p}+y^{p}}\right)+\mathcal{T}_{m}^{k} f\left(\sqrt[p]{2 m+1} \sqrt[p]{2 x^{p}-y^{p}}\right)-4 \mathcal{T}_{m}^{k} f\left(\sqrt[p]{2 m+1} \sqrt[p]{x^{p}+y^{p}}\right) \\
& \left.-4 \mathcal{T}_{m}^{k} f\left(\sqrt[p]{2 m+1} \sqrt[p]{x^{p}-y^{p}}\right)-24 \mathcal{T}_{m}^{k} f(\sqrt[p]{2 m+1} x)+6 \mathcal{T}_{m}^{k} f(\sqrt[p]{2 m+1} y) \|_{*}\right\} \\
& \leq \max \left\{\alpha_{m}^{k} h_{1}\left((3 m+2) x^{p}\right) h_{2}\left((3 m+2) y^{p}\right), \alpha_{m}^{k} h_{1}\left((-m) x^{p}\right) h_{2}\left((-m) y^{p}\right),\right. \\
& \alpha_{m}^{k} h_{1}\left((m+1) x^{p}\right) h_{2}\left((m+1) y^{p}\right), \alpha_{m}^{k} h_{1}\left((4 m+3) x^{p}\right) h_{2}\left((4 m+3) y^{p}\right), \\
& \left.\alpha_{m}^{k} h_{1}\left((2 m+1) x^{p}\right) h_{2}\left((2 m+1) y^{p}\right)\right\} \\
& \leq \alpha_{m}^{k} h_{1}\left(x^{p}\right) h_{2}\left(y^{p}\right) \max \left\{\lambda_{1}(3 m+2) \lambda_{2}(3 m+2), \lambda_{1}(-m) \lambda_{2}(-m),\right. \\
& \left.\lambda_{1}(m+1) \lambda_{2}(m+1), \lambda_{1}(4 m+3) \lambda_{2}(4 m+3), \lambda_{1}(2 m+1) \lambda_{2}(2 m+1)\right\} \\
& =\alpha_{m}^{k+1} h_{1}\left(x^{p}\right) h_{2}\left(y^{p}\right) . \\
&
\end{aligned}
$$

Thus, we have shown that (2.7) holds for $n \in \mathbf{N}_{0}$, and all $m \in \mathcal{U}$. Letting $n \rightarrow \infty$ in (2.7), we obtain

$$
\begin{aligned}
& \mathcal{F}_{m}\left(\sqrt[p]{2 x^{p}+y^{p}}\right)+\mathcal{F}_{m}(\left.\sqrt[p]{2 x^{p}-y^{p}}\right)=4 \mathcal{F}_{m}\left(\sqrt[p]{x^{p}+y^{p}}\right)+4 \mathcal{F}_{m}\left(\sqrt[p]{x^{p}-y^{p}}\right) \\
&+24 \mathcal{F}_{m}(x)-6 \mathcal{F}_{m}(y)
\end{aligned}
$$

for all $x, y \in \mathbf{R}_{0}$ such that $x \neq y, x \neq-y, y \neq \sqrt[p]{2} x, y \neq-\sqrt[p]{2} x$ and $m \in \mathcal{U}$. This implies that $\mathcal{F}_{m}: \mathbf{R} \rightarrow X$ is a solution of the equation (1.4). Therefore, we construct a sequence $\left\{\mathcal{F}_{m}\right\}_{m \in \mathcal{U}}$ of the solutions of equation (1.4) on $\mathbf{R}_{0}$ such that 


$$
\begin{aligned}
\left\|\mathcal{F}_{m}(x)-f(x)\right\|_{*} & \leq \sup _{n \in \mathbf{N}} \Lambda_{m}^{n} \varepsilon_{m}(x) \\
& \leq \sup _{n \in \mathbf{N}}\left\{\lambda_{1}(m+1) \lambda_{2}(2 m+1) \alpha_{m}^{n} h_{1}\left(x^{p}\right) h_{2}\left(x^{p}\right)\right\},
\end{aligned}
$$

for all $x \in \mathbf{R}_{0}$ and all $m \in \mathcal{U}$. Letting $n \rightarrow \infty$ in the previous inequality and using (2.1), we deduce that $f$ is a solution of the equation (1.4) on $\mathbf{R}_{0}$ which means that the equation (1.4) is hyperstable on $\mathbf{R}_{0}$.

In a similar way, we can prove the following theorem.

Theorem 2.3. Let $p$ be an odd natural number, $\left(X,\|\cdot\|_{*}\right)$ be a nonArchimedean Banach space and let $h: \mathbf{R}_{0} \rightarrow \mathbf{R}_{+}$be a mapping such that

$$
\begin{aligned}
\mathcal{U}: & =\left\{n \in \mathbf{N}: \alpha_{n}=\max \{\lambda(3 n+2), \lambda(-n), \lambda(n+1), \lambda(4 n+3),\right. \\
& \lambda(2 n+1)\}<1\} \neq \phi,
\end{aligned}
$$

where

$$
\lambda(n)=\inf \left\{t \in \mathbf{R}_{+}: h(n x) \leq t h(x), \quad x \in \mathbf{R}_{0}\right\},
$$

for all $n \in \mathbf{N}$, such that

$$
\lim _{n \rightarrow \infty}(\lambda(n+1)+\lambda(2 n+1))=0 .
$$

Assume that $f: \mathbf{R} \rightarrow X$ satisfies the inequality

$$
\begin{aligned}
& \| f\left(\sqrt[p]{2 x^{p}+y^{p}}\right)+f\left(\sqrt[p]{2 x^{p}-y^{p}}\right)-4 f\left(\sqrt[p]{x^{p}+y^{p}}\right)-4 f\left(\sqrt[p]{x^{p}-y^{p}}\right) \\
& -24 f(x)+6 f(y) \|_{*} \leq h\left(x^{p}\right)+h\left(y^{p}\right),
\end{aligned}
$$

for all $x, y \in \mathbf{R}_{0}$ such that $x \neq y, \quad x \neq-y, \quad y \neq \sqrt[p]{2} x$ and $y \neq-\sqrt[p]{2} x$. Then $f$ is a solution of the equation (1.4) on $\mathbf{R}_{0}$.

Proof. We will suffice with the basic idea of the proof. Replacing $x$ by $\sqrt[p]{m+1} x$ and $y$ by $\sqrt[p]{2 m+1} x$ in the inequality (2.8) where $x \in \mathbf{R}_{0}, m \in$ $\mathcal{U}$, we get

$$
\begin{aligned}
& \| 4 f(\sqrt[p]{3 m+2} x)+4 f(\sqrt[p]{-m} x)+24 f(\sqrt[p]{m+1} x)-f(\sqrt[p]{4 m+3} x) \\
& -6 f(\sqrt[p]{2 m+1} x)-f(x) \|_{*} \leq h\left((m+1) x^{p}\right)+h\left((2 m+1) x^{p}\right) \\
& \leq(\lambda(m+1)+\lambda(2 m+1)) h\left(x^{p}\right),
\end{aligned}
$$


for all $m \in \mathcal{U}$ and all $x \in \mathbf{R}_{0}$. We define operators $\mathcal{T}_{m}: X^{\mathbf{R}_{0}} \rightarrow X^{\mathbf{R}_{0}}$ and $\Lambda_{m}: \mathbf{R}_{+}^{\mathbf{R}_{0}} \rightarrow \mathbf{R}_{+}^{\mathbf{R}_{0}}$ by

$$
\begin{aligned}
\mathcal{T}_{m} \xi(x):=4 \xi(\sqrt[p]{3 m+2} x) & +4 \xi(\sqrt[p]{-m} x)+24 \xi(\sqrt[p]{m+1} x)-\xi(\sqrt[p]{4 m+3} x) \\
& -6 \xi(\sqrt[p]{2 m+1} x),
\end{aligned}
$$

for all $\xi \in X^{\mathbf{R}_{0}}$ and all $x \in \mathbf{R}_{0}$ and

$$
\begin{gathered}
\Lambda_{m} \delta(x):=\max \{\delta(\sqrt[p]{3 m+2} x), \delta(\sqrt[p]{-m} x), \delta(\sqrt[p]{m+1} x), \delta(\sqrt[p]{4 m+3} x), \\
\delta(\sqrt[p]{2 m+1} x)\} .
\end{gathered}
$$

Moreover, we write

$\varepsilon_{m}(x)=h\left((m+1) x^{p}\right)+h\left((2 m+1) x^{p}\right) \leq(\lambda(m+1)+\lambda(2 m+1)) h\left(x^{p}\right), \quad x \in \mathbf{R}_{0}$.

As in Theorem 2.2, we observe that inequality (2.8) takes the following form

$$
\left\|f(x)-\mathcal{T}_{m}(x) \mid\right\|_{*} \leq \varepsilon_{m}(x), \quad x \in \mathbf{R}_{0}, m \in \mathcal{U},
$$

then we complete the proof by similar steps of the proof of Theorem 2.2.

\section{Consequences}

In this section, we get, as particular cases of our main results, the hyperstability results in the sens of Hyers-Ulam-Rassiass. Also, we get the same results for the inhomogeneous general $p$-radical functional equation

$$
\begin{aligned}
& f\left(\sqrt[p]{2 x^{p}+y^{p}}\right)+f\left(\sqrt[p]{2 x^{p}-y^{p}}\right)=4 f\left(\sqrt[p]{x^{p}+y^{p}}\right)+4 f\left(\sqrt[p]{x^{p}-y^{p}}\right)+24 f(x) \\
& -6 f(y)+G(x, y) .
\end{aligned}
$$

Corollary 3.1. Let $p$ be an odd natural number, $\left(X,\|\cdot\|_{*}\right)$ be a nonArchimedean Banach space and let $c, r, s \in \mathbf{R}$ such that $r+s<0$ and $c \geq 0$. Assume that a function $f: \mathbf{R} \rightarrow X$ satisfies the inequality

$$
\begin{aligned}
& \| f\left(\sqrt[p]{2 x^{p}+y^{p}}\right)+f\left(\sqrt[p]{2 x^{p}-y^{p}}\right)-4 f\left(\sqrt[p]{x^{p}+y^{p}}\right)-4 f\left(\sqrt[p]{x^{p}-y^{p}}\right)-24 f(x) \\
& +6 f(y) \|_{*} \leq c\left|Q_{1}\left(x^{p}\right)\right|^{r}\left|Q_{2}\left(y^{p}\right)\right|^{s}
\end{aligned}
$$


for all $x, y \in \mathbf{R}_{0}$ where $Q_{1}, Q_{2}: \mathbf{R} \rightarrow \mathbf{R}_{+}$are two quartic mappings. Then $f$ is a solution of the equation (1.4) on $\mathbf{R}_{0}$.

Proof. The proof follows from Theorem 2.2 by taking $h_{1}, h_{2}: \mathbf{R}_{0} \rightarrow \mathbf{R}_{+}$ as follows:

$$
h_{1}\left(x^{p}\right)=c_{1}\left|Q_{1}\left(x^{p}\right)\right|^{r}
$$

and

$$
h_{2}\left(x^{p}\right)=c_{2}\left|Q_{2}\left(x^{p}\right)\right|^{s}
$$

for all $x, y \in \mathbf{R}_{0}$ where $c_{1}, c_{2} \in \mathbf{R}_{+}$such that $c_{1} c_{2}=c \geq 0$. For each $m \in \mathbf{N}$, we define $\lambda_{1}(m)$ as in Theorem 2.2 by

$$
\begin{aligned}
\lambda_{1}(m) & =\inf \left\{t \in \mathbf{R}_{+}: h_{1}\left(m x^{p}\right) \leq t h_{1}\left(x^{p}\right)\right\} \\
& =\inf \left\{t \in \mathbf{R}_{+}: c_{1}\left|Q_{1}\left(m x^{p}\right)\right|^{r} \leq t c_{1}\left|Q_{1}\left(x^{p}\right)\right|^{r}\right\} \\
& =\inf \left\{t \in \mathbf{R}_{+}: m^{4 r}\left|Q_{1}\left(x^{p}\right)\right|^{r} \leq t\left|Q_{1}\left(x^{p}\right)\right|^{r}\right. \\
& =m^{4 r},
\end{aligned}
$$

for all $x \in \mathbf{R}_{0}$. Also, for each $m \in \mathbf{N}$, we have $\lambda_{2}(m)=m^{4 s}$. It is clear that there exists $m_{0} \in \mathbf{N}$ such that, for each $m \geq m_{0}$, we get

$$
\begin{aligned}
\alpha_{m}= & \max \left\{\lambda_{1}(3 m+2) \lambda_{2}(3 m+2), \lambda_{1}(-m) \lambda_{2}(-m),\right. \\
& \lambda_{1}(m+1) \lambda_{2}(m+1), \\
& \left.\lambda_{1}(4 m+3) \lambda_{2}(4 m+3), \lambda_{1}(2 m+1) \lambda_{2}(2 m+1)\right\}, \\
= & \max \left\{(3 m+2)^{4(r+s)},(-m)^{4(r+s)},(m+1)^{4(r+s)},\right. \\
& \left.(4 m+3)^{4(r+s)},(2 m+1)^{4(r+s)}\right\}<1
\end{aligned}
$$

According to Theorem 2.2, there exists a unique function $\mathcal{F}_{m}: \mathbf{R}_{0} \rightarrow X$ satisfies the equation (1.4) such that

$$
\begin{aligned}
\left\|\mathcal{F}_{m}-f(x)\right\|_{*} & \leq c \sup _{n \in \mathbf{N}}\left\{\lambda_{1}(m+1) \lambda_{2}(2 m+1) \alpha_{m}^{n}\left|Q_{1}\left(x^{p}\right)\right|^{r}\left|Q_{2}\left(x^{p}\right)\right|^{s}\right\} \\
& =c(m+1)^{4 r}(2 m+1)^{4 s}\left|Q_{1}\left(x^{p}\right)\right|^{r}\left|Q_{2}\left(x^{p}\right)\right|^{s} \sup _{n \in \mathbf{N}}\left\{\alpha_{m}^{n}\right\},
\end{aligned}
$$

for all $x \in \mathbf{R}_{0}$. On the other hand, Since $r+s<0$, one of $r, s$ must be negative. Assume that $r<0$. Then

$$
\lim _{m \rightarrow \infty} \lambda_{1}(m+1) \lambda_{2}(2 m+1)=\lim _{m \rightarrow \infty}(m+1)^{4(r+s)}=0
$$

We get the desired result. 
Corollary 3.2. Let $p$ be an odd natural number, $\left(X,\|\cdot\|_{*}\right)$ be a nonArchimedean Banach space and let $c, r \in \mathbf{R}$ such that $c \geq 0$ and $r<0$. Assume that a function $f: \mathbf{R} \rightarrow X$ satisfies the inequality

$$
\begin{aligned}
& \| f\left(\sqrt[p]{2 x^{p}+y^{p}}\right)+f\left(\sqrt[p]{2 x^{p}-y^{p}}\right)-4 f\left(\sqrt[p]{x^{p}+y^{p}}\right)-4 f\left(\sqrt[p]{x^{p}-y^{p}}\right) \\
& (3.5)-24 f(x)+6 f(y) \|_{*} \\
& \quad \leq c\left(\left|Q\left(x^{p}\right)\right|^{r}+\left|Q\left(y^{p}\right)\right|^{r}\right)
\end{aligned}
$$

for all $x, y \in \mathbf{R}_{0}$ where $Q: \mathbf{R} \rightarrow \mathbf{R}_{+}$is a quartic mapping. Then $f$ is a solution of the equation (1.4) on $\mathbf{R}_{0}$.

Proof. The proof is similar to the proof of Corollary 3.1 with taking $h: \mathbf{R}_{0} \rightarrow \mathbf{R}_{+}$defined by $h\left(x^{p}\right)=c\left|Q\left(x^{p}\right)\right|^{r}$ for all $x \in \mathbf{R}_{0}$ where $c \geq 0$ and $r<0$.

In the following corollaries, we get the hyperstability results for the inhomogeneous general $p$-radical functional equation related to quartic mappings.

Corollary 3.3. Let $p$ be an odd natural number, $c, r, s \in \mathbf{R}$ such that $c \geq 0$ and $r+s<0,\left(X,\|\cdot\|_{*}\right)$ be a non-Archimedean Banach space, $G: \mathbf{R} \times \mathbf{R} \rightarrow X$ be a function such that $G(0,0)=0$ and let $f: \mathbf{R} \rightarrow X$ be a function such that $f(0)=0$. Assume that $f$ and $G$ satisfy the inequality

$$
\begin{aligned}
& \| f\left(\sqrt[p]{2 x^{p}+y^{p}}\right)+f\left(\sqrt[p]{2 x^{p}-y^{p}}\right)-4 f\left(\sqrt[p]{x^{p}+y^{p}}\right)-4 f\left(\sqrt[p]{x^{p}-y^{p}}\right)-24 f(x) \\
& +6 f(y)-G(x, y) \|_{*} \leq c\left|Q_{1}\left(x^{p}\right)\right|^{r}\left|Q_{2}\left(y^{p}\right)\right|^{s}
\end{aligned}
$$

for all $x, y \in \mathbf{R}_{0}$, where $Q_{1}, Q_{2}: \mathbf{R} \rightarrow \mathbf{R}_{+}$are two quartic mappings.If the functional equation

$$
\begin{gathered}
\left(\sqrt[p]{2 x^{p}+y^{p}}\right)+f\left(\sqrt[p]{2 x^{p}-y^{p}}\right)-4 f\left(\sqrt[p]{x^{p}+y^{p}}\right)-4 f\left(\sqrt[p]{x^{p}-y^{p}}\right)-24 f(x) \\
+6 f(y)-G(x, y)=0
\end{gathered}
$$

has a solution $f_{0}: \mathbf{R} \rightarrow X$ on $\mathbf{R}_{0}$, then $f$ is a solution of the equation (3.6) on $\mathbf{R}_{0}$. 
Proof. Let $\psi: \mathbf{R} \rightarrow X$ be a function defined by $\psi(x):=f(x)-f_{0}(x)$ for all $x \in \mathbf{R}$. Then we get that

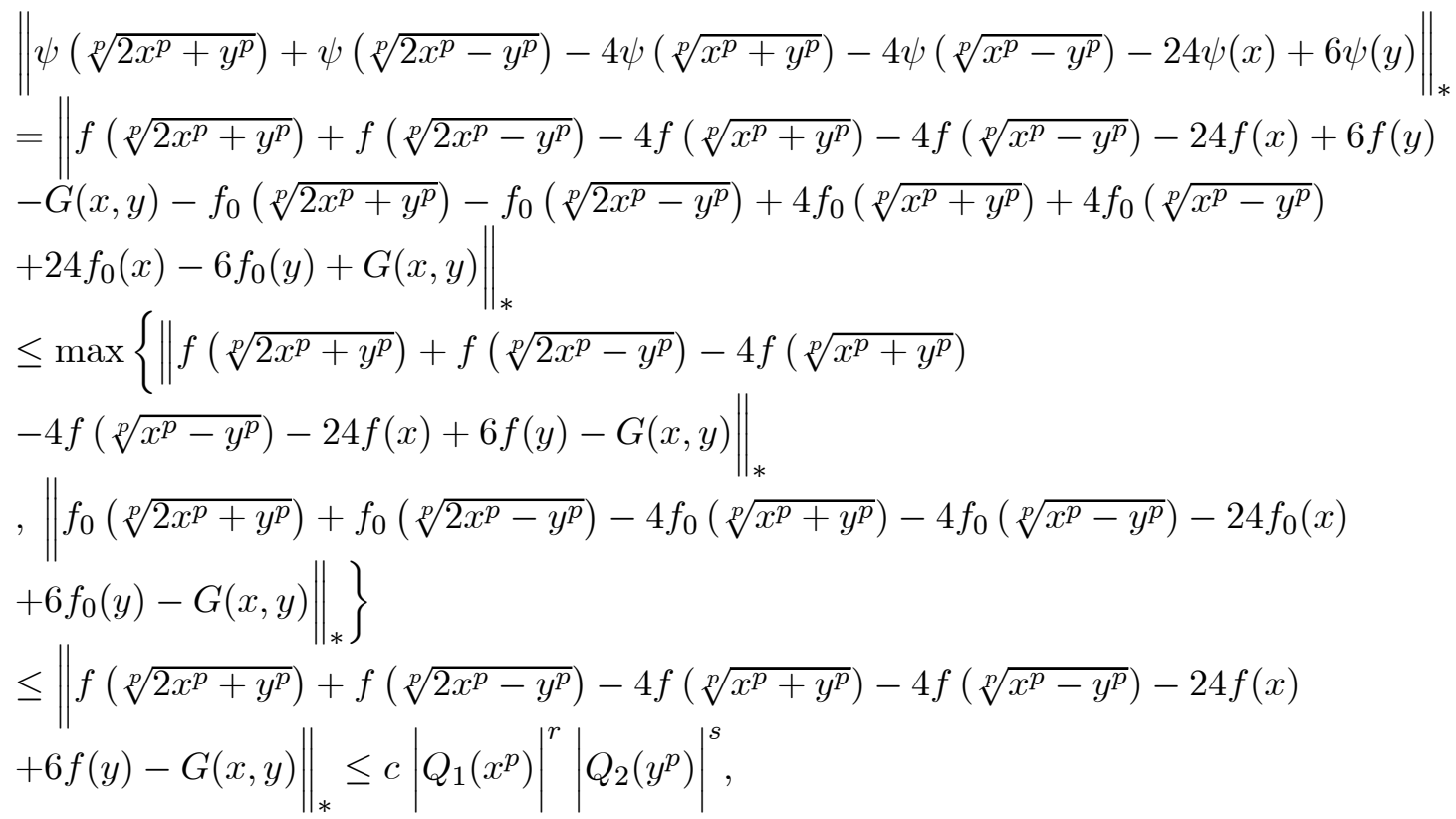

for all $x, y \in \mathbf{R}_{0}$. By using Corollary 3.1, we deduce that $\psi$ is a solution of equation (1.4). Moreover, for all $x, y \in \mathbf{R}_{0}$, we have

$$
\begin{aligned}
& f\left(\sqrt[p]{2 x^{p}+y^{p}}\right)+f\left(\sqrt[p]{2 x^{p}-y^{p}}\right)-4 f\left(\sqrt[p]{x^{p}+y^{p}}\right)-4 f\left(\sqrt[p]{x^{p}-y^{p}}\right) \\
& -24 f(x)+6 f(y)-G(x, y) \\
& =\psi\left(\sqrt[p]{2 x^{p}+y^{p}}\right)+\psi\left(\sqrt[p]{2 x^{p}-y^{p}}\right)-4 \psi\left(\sqrt[p]{x^{p}+y^{p}}\right)-4 \psi\left(\sqrt[p]{x^{p}-y^{p}}\right) \\
& -24 \psi(x)+6 \psi(y)+f_{0}\left(\sqrt[p]{2 x^{p}+y^{p}}\right)+f_{0}\left(\sqrt[p]{2 x^{p}-y^{p}}\right) \\
& -4 f_{0}\left(\sqrt[p]{x^{p}+y^{p}}\right)-4 f_{0}\left(\sqrt[p]{x^{p}-y^{p}}\right)-24 f_{0}(x)+6 f_{0}(y)-G(x, y)=0
\end{aligned}
$$

which means that $f$ is a solution of (3.6) on $\mathbf{R}_{0}$.

With an analogous proof of Corollary 3.3, we can prove the following corollary.

Corollary 3.4. Let $p$ be an odd natural number, $c, r \in \mathbf{R}$ such that $c \geq 0$ and $r<0,\left(X,\|\cdot\|_{*}\right)$ be a non-Archimedean Banach space and let $G$ : $\mathbf{R} \times \mathbf{R} \rightarrow X$ be a function such that $G(0,0)=0$ and $f: \mathbf{R} \rightarrow X$ be a function such that $f(0)=0$. Assume that $f$ and $G$ satisfy the inequality 


$$
\begin{aligned}
& \| f\left(\sqrt[p]{2 x^{p}+y^{p}}\right)+f\left(\sqrt[p]{2 x^{p}-y^{p}}\right)-4 f\left(\sqrt[p]{x^{p}+y^{p}}\right)-4 f\left(\sqrt[p]{x^{p}-y^{p}}\right)-24 f(x) \\
& +6 f(y)-G(x, y) \|_{*} \leq c\left(\left|Q\left(x^{p}\right)\right|^{r}+\left|Q\left(y^{p}\right)\right|^{r}\right)
\end{aligned}
$$

for all $x, y \in \mathbf{R}_{0}$, where $Q: \mathbf{R} \rightarrow \mathbf{R}_{+}$is a quartic mapping. If the functional equation

$f\left(\sqrt[p]{2 x^{p}+y^{p}}\right)+f\left(\sqrt[p]{2 x^{p}-y^{p}}\right)-4 f\left(\sqrt[p]{x^{p}+y^{p}}\right)-4 f\left(\sqrt[p]{x^{p}-y^{p}}\right)-24 f(x)$ $+6 f(y)-G(x, y)=0$,

has a solution $f_{0}: \mathbf{R} \rightarrow X$ on $\mathbf{R}_{0}$, then $f$ is a solution of the equation (3.8) on $\mathbf{R}_{0}$.

\section{References}

[1] L. Aiemsomboon and W. Sintunavarat, "On a new type of stability of a radical quadratic functional equation using Brzdȩk's fixed point theorem", Acta Mathematica Hungarica, vol. 151, no. 1, pp. 35-46, 2016, doi: 10.1007/ s10474-016-0666-2

[2] L. Aiemsomboon and W. Sintunavarat, "On generalized hyperstability of a general linear equation", Acta Mathematica Hungarica, vol. 149, no. 2, pp. 413- 422, 2016, doi: 10.1007/s10474-016-0621-2

[3] M. Almahalebi, "Stability of a generalization of Cauchy's and the quadratic functional equations", Journal of Fixed Point Theory and Applications, vol. 20, no. 12, 2018, doi:10.1007/ s11784-018-0503-z

[4] M. Almahalebi, A. Charifi, and S. Kabbaj, "Hyperstability of a Cauchy functional equation", Journal of Nonlinear Analysis and Optimization: Theory Applications, vol. 6, no. 2, pp. 127-137, 2015.

[5] M. Almahalebi and C. Park, "On the hyperstability of a functional equation in commutative groups", Journal of Computational Analysis Applications, vol. 20, no. 1, pp. 826-833, 2016. 
[6] M. Almahalebi and A. Chahbi, "Hyperstability of the Jensen functional equation in ultrametric spaces", Aequationes mathematicae, vol. 91, no. 4, pp. 647661, 2017.

[7] M. Almahalebi and A. Chahbi, "Approximate solution of p-radical functional equation in 2-Banach spaces", Acta Mathematica Scientia, vol. 39, no. 2, pp. 551-566, 2019.

[8] Z. Alizadeh and A. G. Ghazanfari, "On the stability of a radical cubic functional equation in quasi- $\beta$-spaces", Journal of Fixed Point Theory and Applications, vol. 18, Art ID. 843, 2016, doi: 10.1007/ s11784-016-0317-9

[9] T. Aoki, "On the stability of the linear transformation in Banach spaces", Journal of Mathematical Society of Japan, vol. 2, pp. 64-66, 1950.

[10] D. G. Bourgin, "Classes of transformations and bordering transformations", Bulletin of the American Mathematical Society, vol. 57, pp. 223-237, 1951.

[11] N. Brillouët-Belluot, J. Brzdȩk, and K. Ciepliński, “On some recent developments in Ulam's type stability", Abstract and Applied Analysis, vol. 2012, Art. ID 716936, 2012, doi:10.1155/ 2012/ 716936

[12] J. Brzdęk and J. Tabor, "A note on stability of additive mappings", in Stability of mappings of Hyers-Ulam type, T. M. Rassias, Ed. Palm Harbor, FL: Hadronic Press, 1994, pp. 19-22.

[13] J. Brzdęk, J. Chudziak and Z. Páles, "A fixed point approach to stability of functional equations", Nonlinear Analysis: Theory, Methods \& Applications, vol. 74, no.17, pp. 6728-6732, 2011.

[14] J. Brzdȩk and K. Ciepliński, "A fixed point approach to the stability of functional equations in non-Archimedean metric spaces", Nonlinear Analysis: Theory, Methods \& Applications, vol. 74, no. 18, pp. 6861-6867, 2011.

[15] J. Brzdęk, "Stability of additivity and fixed point methods", Fixed Point Theory and Applications, vol. 2013, Art. ID. 265, 2013, doi: 10.1186/ 1687-1812-2013-285

[16] J. Brzdęk, "Hyperstability of the Cauchy equation on restricted domains", Acta Mathematica Hungarica, vol. 141, pp. 58-67, 2013.

[17] J. Brzdẹk, L. Cădariu, and K. Ciepliński, "Fixed point theory and the Ulam stability", Journal of Function Spaces, vol. 2014, Art. ID 829419, 2014, doi: 10.1155/2014/ 829419 
[18] J. Brzdęk, W. Fechner, M. S. Moslehian and J. Sikorska, "Recent developments of the conditional stability of the homomorphism equation", Banach Journal of the Mathematical Analysis, vol. 9, no. 3, pp. 278-327, 2015.

[19] J. Brzdęk, "Remark 3", Annales Universitatis Paedagogicae Cracoviensis Studia Mathematica, vol. 14, p. 196, 2015.

[20] J. Brzdęk, "Remarks on solutions to the functional equations of the radical type", Advances in the Theory of Nonlinear Analysis and its Application, vol. 1, pp. 125-135, 2017.

[21] M. Eshaghi Gordji and M. Parviz, "On the Hyers Ulam stability of the functional equation $f\left(\sqrt[2]{x^{2}+y^{2}}\right)=f(x)+f(y)$ ", Nonlinear functional analysis and applications, vol. 14, pp. 413-420, 2009.

[22] M. Eshaghi Gordji, H. Khodaei, A. Ebadian and G. H. Kim, "Nearly radical quadratic functional equations in p-2-normed spaces", Abstract and Applied Analysis, vol 2012, Art. ID 896032, 2012, doi: 10.1155/2012/ 896032

[23] S. Gähler, "2-metrische Räume und ihre topologische Struktur", Mathematische Nachrichten, vol. 26, pp. 115-148, 1963.

[24] S. Gähler, "Linear 2-normiete Räumen”, Mathematische Nachrichten, vol. 28, pp. 1-43, 1964.

[25] P. Găvruţa, "A generalization of the Hyers-Ulam-Rassias stability of approximately additive mappings", Journal of Mathematical Analysis and Applications, vol. 184, pp. 431-436, 1994.

[26] D. H. Hyers, "On the stability of the linear functional equation", Proceedings of the National Academy of Sciences, vol. 27, no. 4, pp. 222-224, 1941.

[27] H. Khodaei, M. Eshaghi Gordji, S. S. Kim, and Y. J. Cho, "Approximation of radical functional equations related to quadratic and quartic mappings", Journal of Mathematical Analysis and Applications, vol. 395, pp. 284-297, 2012.

[28] S. S. Kim, Y. J. Cho, and M. Eshaghi Gordji, "On the generalized HyersUlam-Rassias stability problem of radical functional equations", Journal of Inequalities and Applications, vol. 2012, Art. ID. 186, 2012, doi: 10.1186/ 1029-242X-2012-186 
[29] S. H. Lee, S. M. Im, and I. S. Hwang, "Quartic functional equations", Journal of Mathematical Analysis and Applications, vol. 307, no. 2, 387-394, 2005.

[30] G. Maksa and Z. Pales, "Hyperstability of a class of linear functional equations", Acta Mathematica Academiae Paedagogicae Nyiregyhaziensis, vol. 17, no. 2, pp. 107-112, 2001.

[31] W. -G. Park, "Approximate additive mappings in 2-Banach spaces and related topics", Journal of Mathematical Analysis and Applications, vol. 376, no. 1, pp. 193-202, 2011.

[32] "16th International Conference on Functional Equations and Inequalities, Będlewo, Poland, May 17-23, 2015 Report of Meeting", Annales Universitatis Paedagogicae Cracoviensis Studia Mathematica, vol. 14, pp. 163-202, 2015.

[33] T. M. Rassias, "On the stability of the linear mapping in Banach spaces", Proceedings of the American Mathematical Society, vol. 72, no. 2, pp. 297-300, 1978.

[34] T. M. Rassias, "Problem 16; 2", Aequationes mathematicae, vol. 39, pp. 292-293, 1990.

[35] T. M. Rassias, "On a modified Hyers-Ulam sequence”, Journal of Mathematical Analysis and Applications, vol. 158, pp. 106-113, 1991.

[36] S. M. Ulam, Problems in Modern Mathematics. New York (NY): JohnWiley \& Sons Inc., 1964. 


\author{
Ahmed Nuino \\ Department of Mathematics, \\ Faculty of Sciences, \\ University of Ibn Tofail, \\ B. P. 133 Kenitra, \\ Morocco \\ e-mail: ahmed.nuino2015@gmail.com \\ Corresponding author \\ Mustapha Esseghyr Hryrou \\ Department of Mathematics, \\ Faculty of Sciences, \\ University of Ibn Tofail, \\ B. P. 133 Kenitra, \\ Morocco \\ e-mail: hryrou.mustapha@hotmail.com \\ and

\section{Samir Kabbaj} \\ Department of Mathematics, \\ Faculty of Sciences, \\ University of Ibn Tofail, \\ B. P. 133 Kenitra, \\ Morocco \\ e-mail: samkabbaj@yahoo.fr
}

